\title{
Enhanced corticospinal response to observed pain in pain synesthetes
}

\author{
Bernadette M. Fitzgibbon • Peter G. Enticott • \\ John L. Bradshaw • Melita J. Giummarra • \\ Michael Chou • Nellie Georgiou-Karistianis • \\ Paul B. Fitzgerald
}

Published online: 27 December 2011

(C) Psychonomic Society, Inc. 2011

\begin{abstract}
Observing noxious injury to another's hand is known to induce corticospinal inhibition that can be measured in the observer's corresponding muscle. Here, we investigated whether acquired pain synesthetes, individuals who experience actual pain when observing injury to another, demonstrate less corticospinal inhibition than do controls during pain observation, as a potential mechanism for the experience of vicarious pain. We recorded motor-evoked potentials (MEPs) induced at two time points through transcranial magnetic stimulation while participants observed videos of a hand at rest, a hypodermic needle penetrating the skin, a Q-tip touching the skin, and a hypodermic needle penetrating an apple. We compared MEPs in three groups: 7 amputees who experience pain synesthesia, 11 nonsynesthete amputees who experience phantom limb pain, and 10 healthy controls. Results indicated that the pain synesthete group demonstrated significantly enhanced MEP response
\end{abstract}

B. M. Fitzgibbon · P. G. Enticott • P. B. Fitzgerald Monash Alfred Psychiatry Research Center,

School of Psychology and Psychiatry, Monash University, Old Baker Building, Level One, Alfred Hospital,

Melbourne 3004, Australia

B. M. Fitzgibbon · J. L. Bradshaw $\cdot$ M. J. Giummarra

N. Georgiou-Karistianis

Experimental Neuropsychology Research Unit,

School of Psychology and Psychiatry, Monash University,

Clayton, VIC 3800, Australia

M. Chou

Caufield General Medical Centre, Amputee Unit,

Melbourne, Australia

B. M. Fitzgibbon ( $\triangle)$

Monash Alfred Psychiatry Research Center,

Old Baker Building, The Alfred Hospital,

Melbourne 3004, Australia

e-mail: bernadette.fitzgibbon@monash.edu to the needle penetrating the hand, relative to the needle not having yet penetrated the hand, as compared with controls. This effect was not observed exclusively in the same muscle where noxious stimulation was applied. We speculate that our findings reflect a generalized response to pain observation arising from hyperactivity of motor mirror neurons not involved in direct one-to-one simulation but, rather, in the representation of another's experience.

Keywords Synesthesia $\cdot$ Synesthetic pain · Phantom limb pain - Empathy for pain - Transcranial magnetic stimulation

\section{Introduction}

The perception of noxious stimulation to another can induce a personal experience of pain. This phenomenon is known as synesthetic pain, an experience that has been described seemingly from birth (congenital; Osborn \& Derbyshire, 2010) and following pain-related trauma (acquired; Fitzgibbon, Enticott, et al., 2010; Giummarra \& Bradshaw, 2008). Early incidence reports of synesthetic pain have suggested that around $30 \%$ of a healthy population experience congenital synesthetic pain and around $16 \%$ of an amputee group report synesthetic pain acquired following amputation (Fitzgibbon, Enticott, et al., 2010). Besides onset, there are key differences between congenital and acquired pain synesthetes: Congenital pain synesthetes experience pain in the same location that they observe injury in another, at an intensity of no more than 3.7/10, and demonstrate higher levels of empathy than do nonsynesthete controls (Osborn \& Derbyshire, 2010). In contrast, acquired pain synesthetes experience high-intensity pain at the site of previous trauma (e.g., the phantom limb; Fitzgibbon, Enticott, et al., 2010) and, according to studies so far, do not demonstrate higher levels of empathy, as compared with 
nonsynesthete controls (Fitzgibbon et al., 2011; Giummarra et al., 2010). The neurobiological mechanisms that underpin synesthetic pain and its variants are currently unknown.

One explanatory model suggests that synesthetic pain may be induced through hyperactivity of vicarious neural circuits, involved in experiencing actual pain and observing noxious stimulation to another (Fitzgibbon, Giummarra, Georgiou-Karistianis, Enticott, \& Bradshaw, 2010b). Vicarious neural activity may occur through mirror neurons, neurons that were first found in the ventral premotor cortex (F5) and the parietal area (PF) of the macaque brain and are active during both action observation and action execution (di Pellegrino, Fadiga, Fogassi, Gallese, \& Rizzolatti, 1992). Although these areas have become known as the classical mirror neuron areas (for a review, see Rizzolatti \& Craighero, 2004), areas of the brain with mirror properties, mirror systems, have since been identified in humans for action (for a review, see Rizzolatti \& Craighero, 2004), as well as for emotions (e.g., Carr, Iacoboni, Dubeau, Mazziotta, \& Lenzi, 2003; Enticott, Johnston, Herring, Hoy, \& Fitzgerald, 2008; Wicker et al., 2003) and for sensations (e.g., Jackson, Meltzoff, \& Decety, 2005; Keysers et al., 2004). It is thought that we understand the actions, emotions, and sensations of others through this mirrored simulation (Rizzolatti, Fogassi, \& Gallese, 2001).

Empathy for pain, the automatic and unconscious perception of pain in another, activates overlapping regions of the brain involved in experiencing actual pain, known as the pain matrix (Peyron, Laurent, \& Garcia-Larrea, 2000; Rainville, 2002). These regions include the primary (S1) and secondary (S2) somatosensory cortices, the insula, and the anterior cingulate cortex (Iannetti \& Mouraux, 2010). Empathy for pain studies have shown that the perception of pain in another activates regions of the pain matrix involved in different aspects of pain, including affective (e.g., Botvinick et al., 2005; Godinho, Magnin, Frot, Perchet, \& Garcia-Larrea, 2006; Jackson et al., 2005; Morrison, Lloyd, di Pellegrino, \& Roberts, 2004; Singer et al., 2004) and sensory (e.g., Avenanti \& Aglioti, 2006; Avenanti, Bueti, Galati, \& Aglioti, 2005; Avenanti, Minio Paluello, Bufalari, \& Aglioti, 2006; Bufalari, Aprile, Avenanti, Di Russo, \& Aglioti, 2007; Cheng, Yang, Lin, Lee, \& Decety, 2008; Yang, Decety, Lee, Chen, \& Cheng, 2009) processing. Critically, the level of activation in affective components of the pain matrix correlates with empathy scores (e.g., Singer et al., 2004), and increased activation of sensory areas correlates with ratings of sensory empathy measured by intensity ratings of observed pain (e.g., Avenanti et al., 2005). Since pain matrix activity overlaps between the experience of actual pain and the observation of pain in another and this activity correlates with behavioral measures, the pain matrix network appears to have mirror properties.
As with other mirror system modalities, cortical activation during empathy for pain is not as great or as widespread as if one was actually experiencing noxious stimulation. It is likely that this reflects inhibitory processes associated with mirror systems (Kraskov, Dancause, Quallo, Shepherd, \& Lemon, 2009) that normally prevent the observer from experiencing pain when observing another experience pain. Considering that pain synesthetes experience pain when seeing others in pain, disruption to inhibitory mechanisms seems plausible as a mechanism underlying this process. That synesthetic pain may come about through the failure of these inhibitory mechanisms has already been suggested by an imaging study of participants who reported synaesthetic pain: People who reported feeling pain when seeing injury in others showed increased activation in more widespread areas of pain-related neural regions when observing pain, as compared with controls (Osborn \& Derbyshire, 2010). In the related experience of synesthetic touch, where seeing another being touched can result in a first-hand tactile sensation to the self, observing touch induces greater and more widespread activation of areas involved in processing actual touch, as compared with nonsynesthete controls (Blakemore, Bristow, Bird, Frith, \& Ward, 2005; Bufalari et al., 2007).

Transcranial magnetic stimulation (TMS) provides a novel way to explore the functioning of mirror systems in response to observed pain. TMS describes a noninvasive method of brain stimulation whereby a magnetic field passes through the scalp, inducing an electrical current altering neural excitability in superficial areas of the brain. When applied to the primary motor cortex (M1), TMS produces an observable motor response in the contralateral extremity muscle, called a motor-evoked potential (MEP). MEPs are thought to reflect corticospinal excitability (CSE), with larger MEPs reflecting a greater number of motor neurones activated by the TMS (Haraldsson, Ferrarelli, Kalin, \& Tononi, 2004), and thus, TMS has been widely used to measure cortical function (e.g., Enticott, Kennedy, Bradshaw, Rinehart, \& Fitzgerald, 2010; Fadiga, Craighero, \& Olivier, 2005; Lepage, Tremblay, \& Theoret, 2010). Using TMS, research investigating empathy for pain response in controls has shown that the observation of pain in another causes a reliable reduction - that is, a pain-related inhibition - in MEP amplitude (Avenanti \& Aglioti, 2006; Avenanti et al., 2005; Avenanti et al., 2006; Avenanti, Minio-Paluello, Sforza, \& Aglioti, 2009; Fecteau, Pascual-Leone, \& Theoret, 2008; Minio-Paluello, Avenanti, \& Aglioti, 2006). This is the same effect that occurs with the application of a painful stimulus to the self, and it is thought to reflect a withdrawal reflex (e.g., Farina, Tinazzi, Le Pera, \& Valeriani, 2003; Le Pera et al., 2001; Svensson, Miles, McKay, \& Ridding, 2003; Urban et al., 2004). Moreover, this MEP reduction has been found to correlate with scores of sensory ratings of the observed pain 
experience (Avenanti et al., 2005) and to occur only in the muscle corresponding to that observed receiving noxious stimulation (e.g., Avenanti et al., 2006; Avenanti, MinioPaluello, Bufalari, \& Aglioti, 2009). Thus, corticospinal inhibition may be a sign of an automatic simulation of another's pain state, linking empathy for pain and the motor system (by way of motor mirror neurons).

There is some evidence to suggest that mirror systems may underlie generalized empathy: the ability to understand another person's state in the context of the self (Decety \& Jackson, 2004; de Vignemont \& Singer, 2006). Support for mirror system involvement in empathy has come from several studies demonstrating a relationship between mirror system activation and self-reported empathy (Gazzola, Aziz-Zadeh, \& Keysers, 2006; Kaplan \& Iacoboni, 2006; Lepage et al., 2010; Pfeifer, Iacoboni, Mazziotta, \& Dapretto, 2008). In one study, participants who scored higher on a measure of empathy showed greater activation in a left-hemispheric temporo-parieto-premotor circuit active during both the execution of an action and when listening to the sound of the same action (Gazzola et al., 2006). In another study, increased activation in the right inferior frontal cortex during the observation of intentional movement was correlated with higher scores on a measure of empathy (Kaplan \& Iacoboni, 2006). However, other studies have not found a relationship between putative mirror system activity and empathy scores (Haker \& Rossler, 2009) or have found a negative relationship between empathy scores and neural activation (Newman-Norlund, Ganesh, van Schie, De Bruijn, \& Bekkering, 2009). If it is true that mirror systems underlie empathy and that mirror systems are hyperactive in pain synesthetes, it could be predicted that pain synesthetes should score higher on measures of empathy, as compared with nonsynesthetes. Indeed, heightened empathy has been found in congenital pain synesthetes (Osborn \& Derbyshire, 2010) and in acquired (Goller, Richards, Novak, \& Ward, 2011) and congenital (Banissy \& Ward, 2007) touch synesthetes. However, this relationship has not been found in acquired pain synesthetes so far (Fitzgibbon et al., 2011; Giummarra et al., 2010).

In the present study, we used TMS to investigate empathy for pain response in amputees who experience synesthetic pain, as compared with controls. To do so, we evaluated CSE of the motor cortex during passive observation of a needle penetrating the hand of a human model. We hypothesized that pain synesthetes would produce less corticospinal inhibition than would controls in response to the observation of pain experienced in another, as seen through an increase in MEP amplitude. We expected this effect to manifest in the muscle congruent to the stimulus observed, implicating mirror system disinhibition in pain synesthetes, but not in nonsynesthetes. Finally, since it is known that interindividual differences may modulate CSE in response to observed pain (e.g., Avenanti, Minio-Paluello, Bufalari, \& Aglioti, 2009), we investigated the relationship between CSE and personal dispositions such as empathy. This allowed us to determine whether amputees who experience synesthetic pain have increased interpersonal characteristics, as has been implicated in congenital pain and touch synesthetes (e.g., Banissy \& Ward, 2007).

\section{Method}

\section{Participants}

Twenty-eight participants were involved in the study. There were three groups: (1) lower-limb amputees who experienced phantom and synesthetic pain (pain synesthetes [PSs]; $n=7$ ); (2) lower-limb amputees who experienced phantom pain, but not synesthetic pain (phantom pain [PP]; $n=11$ ); and (3) nonamputee healthy controls (HCs) who did not experience congenital pain synesthesia $(n=10)$. Healthy controls were recruited through advertisements placed at Monash University and the Alfred Hospital, and amputee participants were invited through the Caulfield General Medical Center or were self-referring from amputee support organizations. Pain synesthete participants were identified if they reported experiencing phantom pain triggered by observing or imaging pain in another. A one-way ANOVA revealed no significant difference between the ages of each group. Chi-square tests for independence revealed no significant difference between cause, or location, of amputation between the amputee groups. However, a significant difference was observed for gender, with the phantom pain group having significantly more males (see Table 1 ). This could not be controlled for, due to difficulty recruiting amputee participants. Participants were excluded if they had a diagnosis of mental illness or neurological condition as verified by self-report, epilepsy (or any history of seizures), a history of serious head injury, or metal in the head (outside of the mouth). Amputee participants were excluded only for mental illnesses other than depression and anxiety (e.g., schizophrenia), due to difficulty in recruitment and the high comorbidity of these disorders in pain populations (see Nicolson, Caplan, Williams, \& Stern, 2009). Informed consent was obtained from all participants prior to commencement of the study. The study was approved by the Monash University Ethics Committee and the Alfred Hospital Ethics Committee.

Visual stimuli

Visual stimuli consisted of four 4-s films showing (1) the right hand at rest (rest), (2) a hypodermic needle penetrating the skin overlying the right FDI muscle (needle), (3) a Q-tip 
Table 1 Demographic variables for all groups
PS, pain synesthete; PP, phantom pain; HC, healthy control

\begin{tabular}{lllll}
\hline & PS & PP & HC & $p$-value \\
\hline N & 7 & 11 & 10 & \\
Age $(M: S D)$ & $55.0(7.79)$ & $49.73(9.79)$ & $54.7(7.38)$ & $.32($ ANOVA) \\
Sex (M:F) & $4: 3$ & $10: 1$ & $4: 6$ & $<.05\left(\chi^{2}\right)$ \\
Cause of amputation & & & & $.39\left(\chi^{2}\right)$ \\
Trauma & 5 & 7 & - & \\
Diabetes/vascular disease & 2 & 1 & - & \\
Cancer & - & 1 & - & $.39\left(\chi^{2}\right)$ \\
Other & - & 2 & - & \\
Location of amputation & & 2 & - & \\
Left leg & 6 & 7 & - & \\
Right leg & 1 & 2 & & \\
Both & - & & & \\
\hline
\end{tabular}

touching the skin overlying the right FDI muscle (Q-tip), or (4) a hypodermic needle penetrating an apple (apple). These stimuli were provided to our group by Dr. Hugo Theoret and were modeled on stimuli previously used to first investigate empathy for pain using TMS (Avenanti et al., 2005) (see Fig. 1).

\section{Procedure}

Participants were seated in a comfortable recliner chair. A 22-in. widescreen (16:9) LCD monitor was positioned at eye level and $120 \mathrm{~cm}$ in front of the participant. EMG was recorded from the right first dorsal interosseus (FDI) and abductor digiti minimi (ADM) muscles. EMG signals were amplified using PowerLab/4SP (AD instruments, Colorado Springs, CO) and were sampled via a CED Micro $1401 \mathrm{mk}$ II analogue-to-digital converting unit (Cambridge Electronic Design, Cambridge U.K.).

Single-pulse TMS was administered using a Magstim 200 stimulator (Magstim Company Ltd., Carmarthenshire, Wales, U.K.) to the left motor cortex (M1) via a hand-held, 70-mm figure-of-eight coil positioned over the scalp. The coil was held above the scalp, with the handle angled backward and $45^{\circ}$ away from the midline. M1 was identified as the location on the scalp able to generate that largest MEP amplitude from the right FDI while at rest. Participants' resting motor threshold (RMT) was defined as the minimum stimulation intensity required to evoke a peak-topeak MEP of $>50 \mu \mathrm{V}$ on at least three out of five consecutive trials (mean RMT $=45.93 \%, S D=6.72$ ).

After the RMT was determined, participants were administered 10 TMS pulses at 125\% RMT at rest (i.e., without any visual stimulus). This was done to obtain a measure of CSE prior to stimulus presentation. Participants then received a series of single TMS pulses (at 125\% RMT) to the left primary motor cortex while watching the video stimuli. The single TMS pulse was delivered at two possible time points (short delay, long delay) following initiation of each film. In the short condition, the TMS pulse was delivered $1 \mathrm{~s}$ after clip initiation, coinciding as the needle or Q-tip was approaching the hand or apple during the dynamic videos. In the long condition, the TMS pulse was delivered $3 \mathrm{~s}$ after clip initiation of the static hand or apple; the latter condition corresponded with when the needle was halfway
Fig. 1 Still images taken from video stimuli according to when TMS pulse was delivered for each delay (short, long)
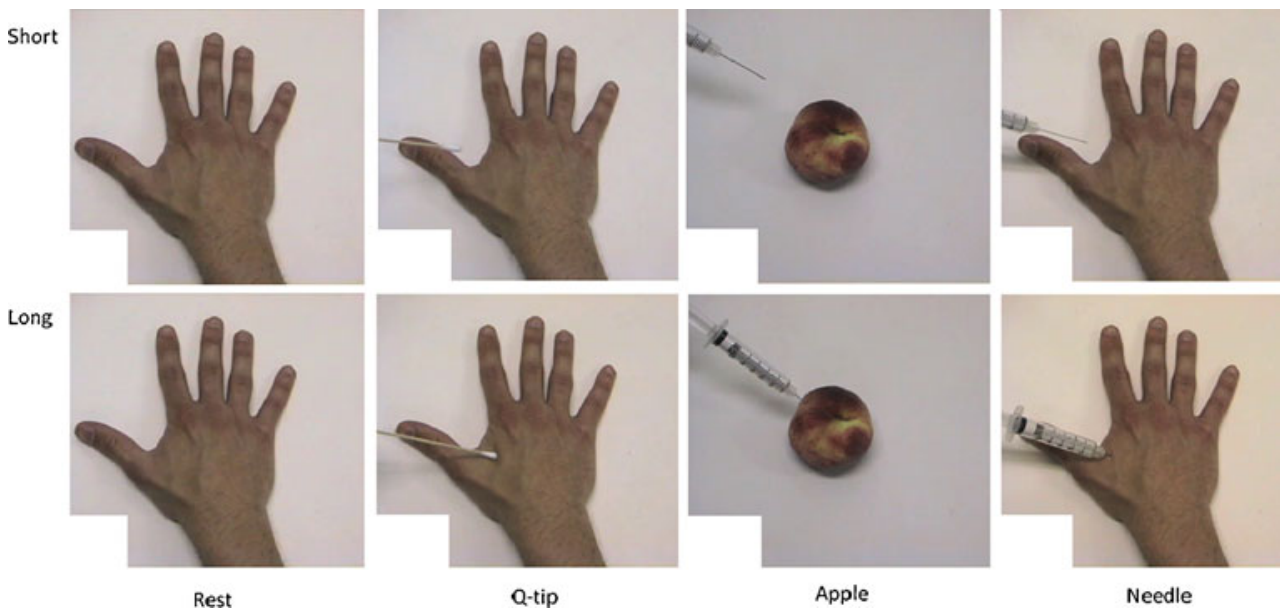
through the skin or apple or when the Q-tip was touching the hand. We expected MEP modulation to be greatest when the needle was penetrating the skin, consistent with previous findings (Avenanti et al., 2006). To trigger the TMS pulse at each time point, a light sensor device was used. To trigger the device, a black square was embedded in the bottom left corner of the video clip, over which the light sensor was placed. When the black square switched briefly $(200 \mathrm{~ms})$ to white, the device sent a trigger (5-V TTL pulse via BNC connector) to the stimulator, thereby sending a trigger to the stimulator to emit a TMS pulse. A second trigger was then sent from the stimulator to the EMG device to signal EMG recording. Overall, one TMS pulse was delivered about every $10 \mathrm{~s}$ (i.e., 6-s gap between each $4 \mathrm{~s}$ of video), with each participant receiving 96 TMS pulses (4 conditions presented 12 times with short delay and another 12 times with a long delay). The videos were presented pseudorandomly across two approximately 6-min blocks, with 48 video stimuli presented in each, with a break of no longer than 2 min between blocks.

Following the video presentations, participants were again administered 10 TMS pulses (125\% RMT) at rest. Since repeated low-frequency (i.e., $\sim 1 \mathrm{~Hz}$ ) pulses can modulate CSE, particularly at intensities above RMT (Fitzgerald, Fountain, \& Daskalakis, 2006), this allowed us to determine whether the TMS procedure itself may have affected our measure of CSE during the empathy for pain component of the experiment.

Finally, participants were asked to complete five questionnaires assessing empathy, anxiety, depression, and pain catastrophization. Empathy was assessed using the Empathy Quotient (EQ; Baron-Cohen \& Wheelwright, 2004) and the Interpersonal Reactivity Index (IRI; Davis, 1980). Anxiety was assessed by the State and Trait Anxiety Inventory (STAI; Spielberger, Gorsuch, \& Lushene, 1970), depression by the Beck Depression Inventory (BDI-II; Beck, Ward, Mendelson, Mock, \& Erbaugh, 1961), and pain catatstrophization by the Pain Catastrophizing Scale (PCS; Sullivan, Bishop, \& Pivik, 1995).

\section{Data analysis}

Individual trials with EMG muscle artifact within $200 \mathrm{~ms}$ prior to the TMS pulse were discarded. Median peak-topeak amplitude $(\mathrm{mV})$ was extracted for each of the video conditions, as well as for the 200-ms period of EMG activity prior to the TMS pulse (root mean square [RMS] amplitude) and for 10 "resting" pulses pre- and post-video-presentation. Median MEP amplitude was selected over mean amplitude for each participant in accordance with the suggestion that TMS measures of CSE may be influenced by an early transitory increase in excitability, which may inaccurately influence the MEP amplitude average (Schmidt et al., 2009).
Data were analyzed using SPSS version 19 (SPSS Inc., Chicago, IL). Data were inspected to ensure adherence to the assumptions of the ANOVA; extreme outliers (3 standard deviations or more) across trials within individual data sets were deleted (fewer than $1 \%$ of all trials). Extreme outliers identified for mean scores within each group were transformed through logarithmic transformation applied to amplitude value ( \pm .1$)$ to normalize data distribution. Before group outliers were removed for MEP amplitude in response to the stimuli, median MEP amplitude for each condition was expressed as a percentage increase (PI), as compared with the rest condition [e.g., (needle rest)/rest $* 100=\mathrm{PI}$ ]. This is consistent with previous research (e.g., Avenanti et al., 2005) and ensures that variance associated with the viewing of a hand is removed, thereby providing a more accurate estimate of mirror system activity (Gangitano, Mottaghy, \& Pascual-Leone, 2001).

A repeated measures mixed model ANOVA with condition (needle, Q-tip, apple), delay (short, long), and muscle (FDI, ADM) as within-subjects factors and group (pain synesthetes, phantom pain, healthy controls) as the between-group factor was run to compare percentage of increase with the rest condition. We also examined what we have termed the needle penetration effect: needle-long relative to needle-short conditions (no other conditions were assessed in this contrast, since this was the only condition to involve actual pain). While previous empathy for pain TMS analysis has executed mixed model ANOVAs looking at percentage change, as compared with rest, it may perhaps be more appropriate to compare needle long with needle short (needle penetration). Thus, this comparison may be a more appropriate control since, in both videos, a needle is present but, in the needle-long condition, the needle is penetrating the skin. To establish the effect of penetration, we ran the following formula: [(PI_NL-PI_NS)/PI_NS] * 100. First, however, we added 100 to each percentage increase, making all values positive, since this equation produced errors when dealing with negative values. We conducted a repeated measures ANOVA with muscle (ADM, FDI) as the within-group factor and group (pain synesthetes, phantom pain, healthy controls) as the between-group factor. Follow-up comparisons were run for all significant effects and were not corrected, due to a small number of comparisons based on prior hypothesis. Partial eta squared $\left(\eta_{\mathrm{p}}{ }^{2}\right)$ was used to determine effect size throughout.

To ensure that tonic muscle activity $200 \mathrm{~ms}$ prior to TMS pulse had no influence on stimulus response, we investigated RMS amplitude by running a repeated measures ANOVA with condition (rest, needle, Q-tip, apple), delay (short, long), and muscle (FDI, ADM) as within-subjects factors and group (pain synesthetes, phantom pain, healthy controls) as the between-group factor. To ensure that there was 
no change in cortical excitability as a result of stimulus presentation, we ran a repeated measures ANOVA on MEP amplitude obtained before and after stimulus presentation, with condition (pre, post) and muscle (ADM, FDI) as within-group factors and group (pain synesthetes, phantom pain, healthy controls) as the between-group factor.

Finally, one-way between-groups ANOVAs were conducted to determine whether there were differences between the groups in scores on the questionnaires. Pearson correlation analyses were then conducted within groups between PI of each of the three videos to rest, and needle suppression amplitude with behavioral questionnaires to investigate whether scores on these measures correlated with MEP modulation. A corrected alpha level of $p<.01$ was set to control for multiple correlations.

\section{Results}

Mean group values for personal dispositional measures are presented in Table 2. Analysis of between-group differences on behavioral measures revealed a significant group difference on the BDI-II, $F(2,27)=3.8, p<.04, \eta_{\mathrm{p}}{ }^{2}=.23$ (see Fig. 4). Post hoc comparison indicated that the phantom pain group had significantly higher scores than the healthy control group $(p=.01)$. No significant differences were observed between the phantom pain and pain synesthete groups $(p=.42)$ or between the healthy control and the pain synesthete groups $(p=.12)$. No other significant differences were observed between groups on measures of empathy, pain catastrophizing, or anxiety.

The repeated measures mixed model ANOVA described above, with group, condition, delay, and muscle as factors and percentage increase from the rest condition as the dependent measure, revealed an effect of condition, $F(2,50)=3.22, p<.05, \eta_{\mathrm{p}}{ }^{2}=.11$. Follow-up comparisons revealed significantly greater MEP amplitudes during the needle condition, as compared with the Q-tip $(p=.04)$, and apple $(p=.03)$ conditions (see Fig. 2). No other main or interaction effects were observed $(p=.07-.88)$.

For needle penetration effect, the repeated measures mixed model ANOVA described above, with muscle and group as factors and percentage increase from the needlelong to the needle-short condition as the dependent measure, revealed a main effect of group, $F(2,25)=4.38, p<.03$, $\eta_{\mathrm{p}}{ }^{2}=.26$. Follow-up comparisons revealed that the pain synesthete group displayed significantly enhanced MEP amplitude, as compared with the healthy control $(p=.02)$ and phantom pain $(p=.02)$ groups. No significant difference was observed between the healthy control and phantom pain groups ( $p=.99$; see Fig. 3 ). No other main or interaction effects were observed $(p=.40-.72)$.

RMS amplitude analysis of the 200 -ms period prior to the TMS pulse revealed no effect of condition on EMG activity, $F(3,75)=1.72, p=.17, \eta_{\mathrm{p}}{ }^{2}=.06$, indicating that the present MEP results cannot be attributed to differences in tonic muscle activity. Comparison of MEP amplitude before and after stimulus presentation revealed no significant effect of condition, $F(1,24)=0.34, p=.57, \eta_{\mathrm{p}}{ }^{2}=.01$, indicating no effects of the TMS procedure on CSE.

Finally, analysis of the relationship of percentage increase values and needle suppression effect with behavioral measures (with an alpha level of $p<.01$ set to control for multiple comparisons) revealed a correlation between needle suppression effect within the FDI muscle in the healthy control group and scores on the PCS, $r=.81, n=10, p=.01$ (see Fig. 4). A near significant relationship was observed between percentage increase of needle long in the FDI
Table 2 Means and $S D$ s of questionnaire scores for each group (HC. healthy controls; PP, phantom pain controls; PS, pain synesthetes)

\begin{tabular}{llll}
\hline & HC $(n=10)$ & PP $(n=11)$ & PS ( $n=7)$ \\
\hline Beck Depression Inventory II & $4.60(4.48)$ & $13.55(9.51)$ & $10.57(7.32)$ \\
State Trait Anxiety Inventory & & & \\
State & $27.90(7.77)$ & $26.27(9.74)$ & $25.57(7.88)$ \\
Trait & $30.80(8.30)$ & $35.82(13.61)$ & $35.71(6.90)$ \\
Pain Catastrophizing Scale & $9.10(8.27)$ & $10.09(9.70)$ & $14.43(9.40)$ \\
Empathy Quotient (15 item Muncer Version) & $17.5(6.75)$ & $17.0(5.22)$ & $16.71(5.15)$ \\
Cognitive Scale & $5.2(2.70)$ & $5.45(2.42)$ & $4.29(2.06)$ \\
Social Scale & $6.20(3.46)$ & $5.91(1.81)$ & $6.0(2.89)$ \\
Emotional Reactivity Scale & $6.10(1.73)$ & $5.64(2.73)$ & $6.43(2.15)$ \\
Interpersonal Reactivity Index & & & \\
Overall Score & $63.00(11.23)$ & $59.73(7.59)$ & $60.00(12.77)$ \\
Perspective Taking Scale & $19.00(5.16)$ & $17.18(4.17)$ & $19.29(2.36)$ \\
Fantasy Scale & $13.20(4.71)$ & $12.73(5.48)$ & $13.71(6.52)$ \\
Empathic Concern Scale & $21.40(5.23)$ & $20.55(3.93)$ & $21.00(3.87)$ \\
Personal Distress Scale & $10.50(5.97)$ & $9.27(3.20)$ & $7.71(3.15)$ \\
\hline
\end{tabular}




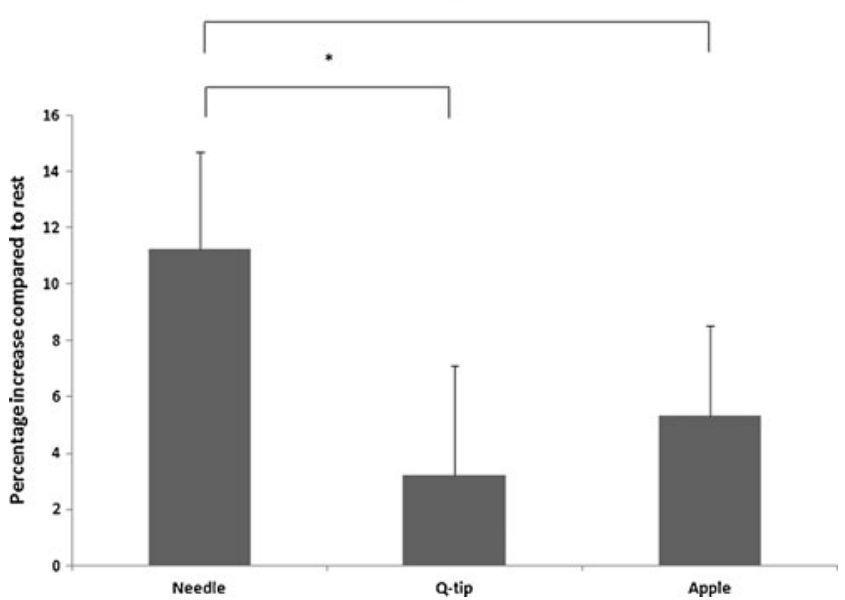

Fig. 2 Percentage increase, with muscle and group combined, for each condition (needle, Q-tip, apple), as compared with rest

muscle and scores on the emotional reactivity subscale of the EQ for the pain synesthete group, $r=.82, n=7, p=.02$ (see Fig. 5).

\section{Discussion}

In the present study, we investigated pain-related inhibition in lower-limb amputees who reported experiencing synesthetic pain, as compared with nonsynesthete lower-limb amputees and nonamputee healthy controls. Previous research has demonstrated that the observation of noxious stimulation to another results in inhibition of CSE in hand muscles (e.g., Avenanti et al., 2005), thought to reflect mirror system activity. Here, we expected that pain synesthetes would produce less inhibition in response to the observation of pain experienced in another, as seen through an increase in MEP amplitude. We found that all participants, regardless of group, delay, or muscle, demonstrated enhanced MEP response to the needle, as compared with the

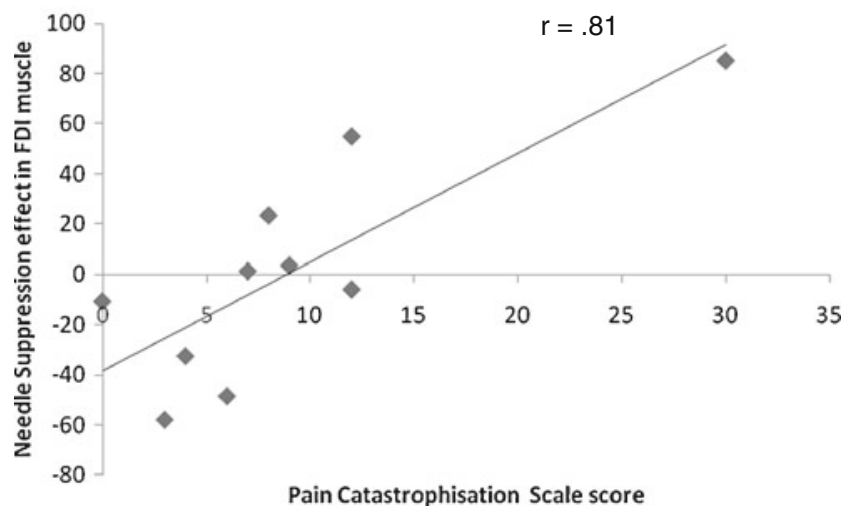

Fig. 4 Correlation between needle suppression effect in the FDI muscle and scores on the Pain Catrophization Scale in the healthy control group

Q-tip or apple condition. This absence of an overall inhibitory response to pain observation does not replicate the results of the prior literature (e.g., Avenanti et al., 2005). It is unclear why we did not find an overall inhibitory response, particularly since there were no obvious methodological differences between this study and previous investigations. It is possible that there is some variability in the reported inhibitory response between individuals, with some individuals showing facilitation, and that we had more of these individuals in the present sample. Mechanisms that may underlie such variation are unknown and warrant future investigation. In support of our hypothesis, however, we found that the pain synesthete group demonstrated enhanced corticospinal facilitation to pain observation, as compared with controls, when the effect of a needle penetrating the skin was compared with that of approaching a static hand (needle penetration effect). We also expected that reduced inhibition in pain synesthetes would be seen in the muscle congruent to the stimulus observed, implicating mirror system properties. Unexpectedly, enhanced facilitation to pain observation in pain synesthetes was seen regardless of muscle and, therefore, was not muscle specific to

Fig. 3 Needle suppression effect in FDI and ADM muscle combined in each group (HC, healthy controls; PP, phantom pain controls; PS, pain synesthetes)
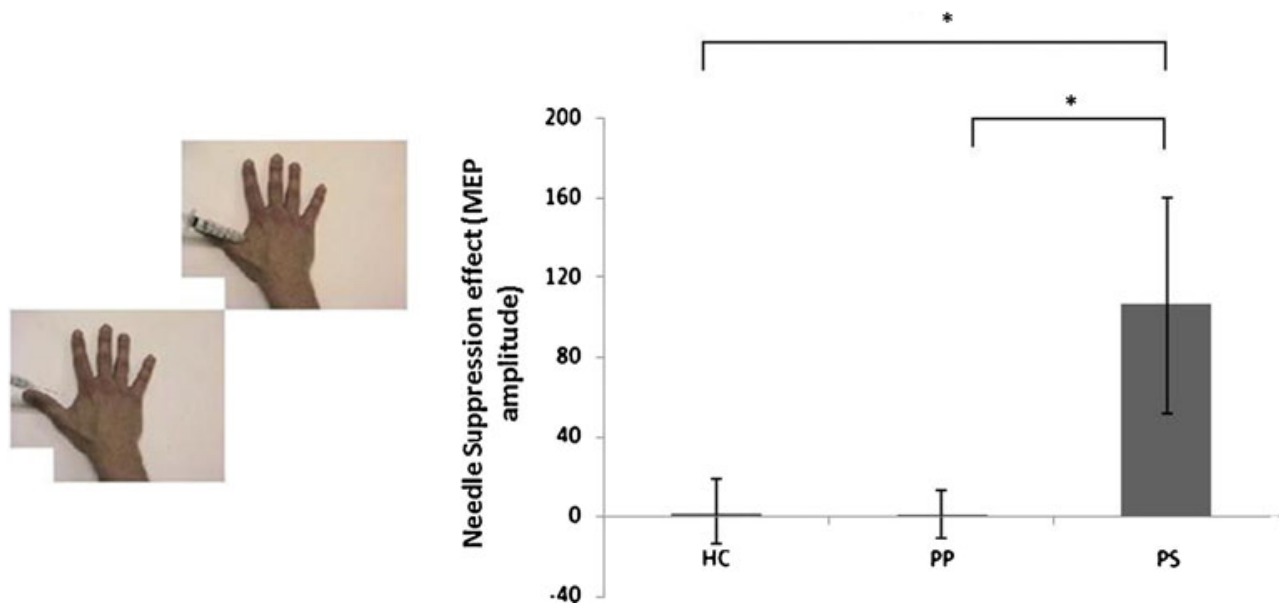


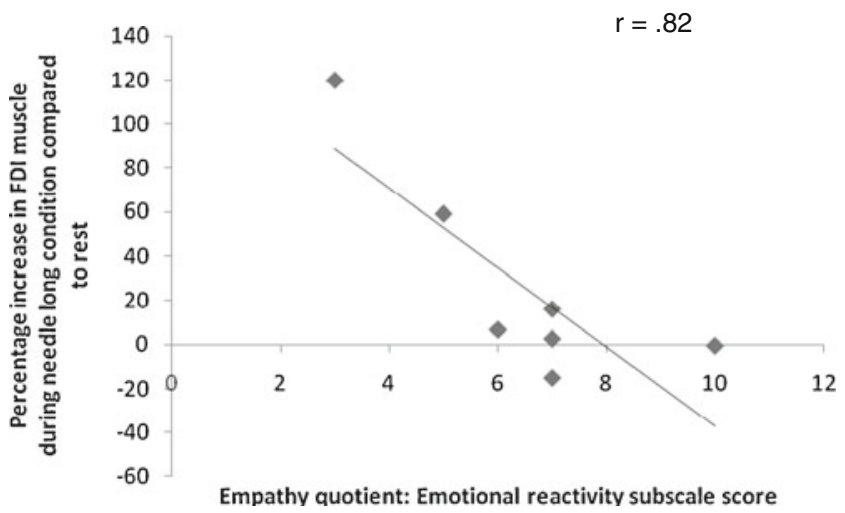

Fig. 5 Correlation between percentage increases during the needlelong condition, as compared with rest, in the FDI muscle and scores on the emotional reactivity subscale of the Empathy Quotient in the pain synesthete group

the site of observed injury (i.e., the FDI muscle). This finding implies facilitation of a more generalized pain response throughout the hand. Since corticospinal activity did not differ before and after stimulus presentation and since there were no differences between groups in tonic muscle activity prior to stimulus presentation, our results cannot be attributed to these factors. Lastly, we investigated whether CSE was related to individual differences in empathy, pain catastrophization, depression, or anxiety. We found that in healthy controls, enhanced MEP response in the needle penetration contrast was correlated only with higher pain catastrophizing scores. We also found that in pain synesthetes, reduced percentage increase during needle penetration was near significantly correlated with higher scores on the emotional reactivity subscale of the empathy quotient.

Enhanced non-muscle-specific response in pain synesthetes

We had hypothesized that pain synesthetes would produce less corticospinal inhibition in response to the observation of pain experienced in another, as seen through an increase in MEP amplitude. This was based on recent research indicating that observing a needle penetrate another's hand brings about a reduced MEP in the observer in a site that is identical to the site of observed noxious injury (Avenanti et al., 2005). This effect has been shown to be site specific and not in a nearby muscle (ADM) that has adjacent motor representations (Krings, Naujokat, \& von Keyserlingk, 1998) or in response to a needle penetrating noncorporeal objects (Avenanti et al., 2005). This inhibitory response is also observed when actual pain is experienced (Farina et al., 2003; Le Pera et al., 2001; Svensson et al., 2003; Urban et al., 2004). Although we did not observe an inhibition effect to pain observation overall, our results indicated that pain synesthetes demonstrate increased facilitation, as compared with controls, in response to pain observation.
Corticospinal inhibition in response to pain observation has been interpreted as indicative of simulation, implicating mirror systems, since it resembles what happens during actual pain stimulation and occurs specifically to the site of observed injury (e.g., Avenanti et al., 2005). Our findings of enhanced MEP response to pain observation in pain synesthetes not only in the specific muscle to which noxious stimulation is applied may reflect a generalized simulated motor facilitation throughout the hand. This generalized motor facilitation may be the result of motor mirror mechanisms that are not effector specific but, rather, involve motor mirror neurons activating a representation of the general area. Indeed, only around $30 \%$ of mirror neurons have been found to be logically related (e.g., di Pellegrino et al., 1992; Gallese, Fadiga, Fogassi, \& Rizzolatti, 1996). These mirror neurons belong to the strictly congruent subtype, being active only when the observed executed action is exactly matched (e.g., reaching for a lever) and when that execution is specific (e.g., the specific grip). Mirror neurons that make up the largest subtype, $60 \%$ of mirror neurons, are known as broadly congruent and are activated during the execution and observation of an action but do so regardless of how the action is carried out (e.g., active in response to any grip). The final mirror neuron subtype, noncongruent, makes up around $10 \%$ of mirror neurons and has no obvious relationship. Accordingly, not all mirror neurons are involved in direct matching (for a discussion of the directmatching hypothesis, see Rizzolatti et al., 2001). Nonspecific activation during empathy for pain has recently been noted - for example, bilateral activation of the somatosensory cortices and activation in response to both painful and nonpainful stimuli (Lamm, Decety, \& Singer, 2011). The absence of direct matching does not imply that mirror neurons, or mirror systems, are not involved in the understanding of others but, rather, suggests that they may often be involved only in a generic representation, not an actual simulation.

Indeed, the reported muscle specificity in pain observation is not all or nothing; in one study, observation of a needle penetrating the FDI muscle induced MEPs recorded in the ADM muscle of healthy controls that trended toward significance for facilitation (Avenanti, Minio-Paluello, Bufalari, \& Aglioti,2009), indicating that the generalized response may simply be less intense, rather than absent. In another study, MEPs were significantly reduced in both the FDI and ADM muscles when the TMS pulse was triggered as the stimuli penetrated or stroked a hand (Fecteau et al., 2008). Avenanti and colleagues (2009a, b) have also demonstrated that pain observation in a hand incongruent with the site of stimulation (e.g., when an injury to a left hand is observed, MEPs are recorded from observers' right hand following stimulation of left M1) induces a generalized hand excitability. In contrast, pain observation congruent with site 
of stimulation elicits the previously reported inhibition suggestive of a freezing response in one hand and an escape response in the other. These findings suggest that the corticospinal pain observation response may not be entirely site specific or purely inhibitory.

Two primary alternative explanations for the implication of mirror system activity in our results may simultaneously explain both our absence of an inhibitory effect to pain stimuli and the increased facilitation in the pain synesthete group, as compared with controls. First, the enhanced activity observed here may reflect increased anticipation of pain to oneself. This is consistent with the suggestion that corticospinal pain-related inhibition may not be exclusive to the processing of actual pain but may reflect somatomotor contagion implicating pain anticipation (Avenanti et al., 2005). This is supported by modulation of pain-related areas of the brain in response to pain anticipation (Ploghaus, Becerra, Borras, \& Borsook, 2003; Porro, Cettolo, Francescato, \& Baraldi, 2003; Wager et al., 2004) and by evidence suggesting that the anticipation of somatosensation can increase activation in the primary somatosensory cortex without actual stimulation (Carlsson, Petrovic, Skare, Petersson, \& Ingvar, 2000). It is plausible that this anticipation may be stronger in pain synesthetes, since observed injury can induce an actual pain experience. However, this possibility is weakened by the absence of an MEP facilitation effect during the short delay where the stimulus approaches the hand.

Our findings may also reflect motor preparation involved in defense mechanisms. Originally, MEP modulation to pain observation was suggested not to indicate a defensive motor reflex, since the inhibition was seen only in the muscle corresponding to that observed receiving injury, and not in a suppression of all hand muscles (Avenanti, MinioPaluello, Bufalari, \& Aglioti, 2009). However, the application of actual pain to an individual involves both inhibitory and facilitatory responses that are not specific to the stimulation site. In one study investigating the effects of electrical stimulation to a digit on MEP amplitudes in distal and proximal upper-limb muscles, inhibitory effects were found predominantly in the distal muscles (Urban et al., 2004). In contrast, facilitatory effects were predominant in proximal muscles. This implicates a protective reflex whereby the painful stimulus is dropped and the hand is withdrawn. Behavioral research also suggests that pain observation may trigger both inhibitory and facilitatory responses that are dependent on the context and imply a defensive/ protective response (Morrison, Poliakoff, Gordon, \& Downing, 2007).

In pain synesthetes, pain observation provides a direct threat; seeing injury to another can cause the experience of pain. It is therefore possible that the non-muscle-specific response seen here in pain synesthetes is a protective strategy whereby facilitation of proximal muscles enables preparation for escape. The increased facilitation in pain synesthetes may reflect greater sensitivity to perceived threat. Although purely speculative, pain inhibition observed in control populations may involve an awareness that the stimuli have no potential to harm them, whereas pain synesthetes are acutely aware that pain observation is the trigger to their synesthetically induced pain. In fact, when an embodied fake or real hand is threatened, lower-limb amputees who report synesthetic pain not only experience pain, but also describe a motor response in the phantom leg (Giummarra et al., 2010). It may be that our findings of increased corticospinal facilitation in pain synesthetes reflects an adaptive motor response to escape from noxious stimuli.

It is worth noting that the absence of an overall corticospinal inhibition effect in each group may be due to movement of the stimuli. It may be more appropriate to deliver a TMS pulse when the stimulus is completely still to avoid movement-induced corticospinal facilitation. Corticospinal facilitation has been observed in response not only to movement (Fecteau et al., 2008), but also to non-pain-related stimuli, including observed touch (Wood, Gallese, \& Cattaneo, 2010), the observation of tool use (Jarvelainen, Schurmann, \& Haria, 2004), mental imagery of movement (Vargas et al., 2004), and implied action (Urgesi, Moro, Candidi, \& Aglioti, 2006).

Modulation of corticospinal excitability and personal dispositions

How pain is experienced can vary greatly between individuals (Coghill, McHaffie, \& Yen, 2003). Similarly, interpersonal differences may modulate the perception of pain experienced in others. Indeed, pain-related MEP inhibition in response to pain observation may be greater in participants who score higher on measures of cognitive empathy and may be reduced in those who score highly for personal distress (Avenanti, Minio-Paluello, Bufalari, \& Aglioti, 2009). In individuals with Asperger's syndrome, MEP modulation in response to pain observation is absent (MinioPaluello, Baron-Cohen, Avenanti, Walsh, \& Aglioti, 2009). These findings suggest that although MEP inhibition during pain observation has been implicated with the processing of sensory pain, nonsensory factors can modulate the effects of observing pain. The effects of interindividual differences have also been observed in a study using magnetoencephalography, where the suppression of somatosensory oscillations correlated with the ability to take on another's perspective (Cheng et al., 2008).

In the present study, we have attempted to account for interpersonal differences by investigating whether, within each group, there would be a relationship between MEP 
response and measures of personal dispositions. After controlling for multiple comparisons, we found only one significant relationship within the healthy control group: Enhanced MEP response in the needle penetration contrast was correlated with higher pain catastrophizing scores. High scores on the PCS have been associated with increased negative pain-related thoughts, emotional distress, and reported pain intensity (Sullivan et al., 1995). This finding may indicate that scores on the pain catastrophization scale may be a good indicator of MEP response to pain observation in healthy controls. Indeed, individuals who score highly for pain catastrophization have been found to report more attention, increased ratings, and negative affect to pain than have low catastrophizers (Van Damme, Crombez, \& Lorenz, 2007; Verhoeven et al., 2010). Thus, pain catastrophization, an affective aspect of pain processing, can modulate the sensorimotor pain effects. Measures of pain catastrophization may not be a good indicator of MEP response in the case of groups with a significant pain history (i.e., amputation). This is particularly evident since PCS scores of the two amputee groups in this study were not significantly different from those for the healthy controls or each other, yet there was no relationship between their PCS scores and MEP modulation. The absence of a relationship between PCS and pain observation in the pain synesthete group also supports the proposition that their experiences of synesthetic pain were not confabulatory or dramatized accounts of otherwise normal pain perception.

We found a near significant relationship between reduced percentage increase during the needle-long condition and higher scores on the emotional reactivity subscale of the empathy quotient in the pain synesthete group. Emotional reactivity is thought to describe the propensity to have an emotional reaction in response to another's state - for example, feeling upset when witnessing another in tears (Lawrence, Shaw, Baker, Baron-Cohen, \& David, 2004). Studies by our group have not previously found any relationship between amputees who report synesthetic pain and measures of empathy (Fitzgibbon et al., 2011; Giummarra et al., 2010). In contrast, touch synesthetes have been found to have greater scores on the emotional reactivity scale than have controls (Banissy \& Ward, 2007). Our results support the possibility that there may be a relationship between pain synesthesia and affective empathy; however, they suggest that higher scores on the emotional reactivity score relate to less MEP modulation in response to pain observation. Further research is warranted to establish more conclusively the nature of this relationship between pain synesthesia and empathy. This should include obtaining measures of sensory empathy, since previous research has indicated that increased MEP modulation has a relationship with higher pain intensity or pain simulation ratings (Avenanti et al., 2005). Even if a positive relationship does exist, increased empathy is most likely a by-product of synesthesia. It would seem unlikely that generalized empathy underlies pain synesthesia, since pain synesthetes experience only observed pain and not a wide range of other experiences - for example, disgust when observing another being disgusted.

\section{Limitations and future directions}

Recruitment of amputees who experience pain synesthesia was difficult and led to a low number of participants. This small sample size may have even prevented identification of possible differences between groups. It is also possible that not all of the recruited pain synesthete participants experienced "true" synesthetic pain. For instance, some people may not feel actual pain when observing pain in others but may experience significant distress that has led them to identify as pain synesthetes. The small sample size may have introduced confounding factors that may have influenced the TMS response, for which we could not control. Gender could not be controlled for, and gender is known to influence empathy for pain processing (e.g., Han, Fan, \& Mao, 2008; Yang et al., 2009). We suggest that our sample size in each of the three groups may also be too small to explore sufficiently the possible influence of personal dispositions on MEP response to pain observation. These potential effects cannot be ruled out, however.

Small sample size may have also influenced the absence of an overall motor inhibition group effect to observing pain, as others have reported (e.g., Avenanti et al., 2005). Inspection of individual data revealed that not all participants in each group displayed the expected inhibition response when observing the pain stimuli. This finding indicates that inhibition in response to pain observation is extremely variable. One factor that may have influenced the absence of inhibition is the timing of TMS pulse delivery. For example, MEP modulation has been found to be greater when needles deeply penetrate rather than pinprick the skin, suggesting that inhibition may be selective to situations perceived to be painful (Avenanti et al., 2006). The absence of consistent inhibition effects should be considered when interpreting the present data, since we cannot compare groups on inhibition in response to pain observation. However, this does not discount the significant differences in muscle facilitation between amputee pain synesthetes, as compared with controls, when observing noxious injury.

Our stimuli may not necessarily have evoked the experience of synesthetic pain in the pain synesthetes. Thus, our study may not compare the experience of actual synesthetic pain with normal pain perception but, rather, may compares a group of people who report synesthetic pain when observing pain in others compared with those who do not. Additionally, only acquired pain synesthetes participated in this 
study, who may differ from what would be found in developmental pain synesthetes.

Stronger stimuli should perhaps be employed in future investigations. In the present study, participants observed a needle penetrating the skin of a still hand. This stimulus may not be sufficiently painful to trigger the effects involved in synesthetic pain. For instance, pain synesthetes typically describe vicarious pain triggered by more intense pain experiences, (e.g., accidents or horror scenes in movies). Alternatively, the experience of synesthetic pain may come about through the processing of stimuli other than the actual noxious injury, such as through facial or auditory pain triggers, or even the real-life context of pain. Subjective data in response to stimuli-for example, ratings of observed pain intensity or unpleasantness-will be useful in future investigations to determine whether pain was felt in response to the stimuli.

Finally, our results relate only to the motor cortex and do not account for the fact that pain, and synesthetic pain specifically, involves additional regions of the pain matrix. Pain perception likely recruits multiple cortical sites that process different aspects of the pain experience. Future potential sites of interest include the prefrontal cortex, associated with the perception of the unpleasantness of pain (Lorenz, Minoshima, \& Casey, 2003), or the parietal lobe, implicated in changes in body perception (e.g., Salanova, Andermann, Rasmussen, Olivier, \& Quesney, 1995) and in the localization of touch and noxious stimuli (Porro et al., 2007). Areas that process the affective and reactive components of the pain experience, such as the anterior cingulate cortex and the insula (Derbyshire et al., 1997; Peyron et al., 2000; Rainville, 2002), should also be explored. Indeed, dysfunction of the insula has been recently identified as a potential mechanism underlying the inability to distinguish between self and other in synesthetic touch (Banissy, Walsh, \& Muggleton, 2011).

\section{Conclusions}

In summary, our results suggest that when observing noxious stimuli, lower-limb amputee pain synesthetes demonstrate enhanced MEP activity, as compared with nonsynesthete amputees and nonamputee healthy controls. This effect was not congruent with the site of observed injury. These findings may represent increased mirror activity in pain synesthetes that is reflective not of one-to-one simulation but of a generalized representation of pain. Alternatively, these findings may implicate increased anticipation for pain or increased readiness, through motor preparation, to execute protective responses. Regardless, our results add to the current literature by demonstrating neurobiological differences in people who report actual pain experience when observing injury. This is a phenomenon that may allow a rare opportunity to investigate pain in the absence of noxious stimulation and social neurobiological mechanisms that underlie empathy for pain.

Acknowledgements David Lee Gow and Susan Thompson at Caulfield General Medical Center. P.B.F. is supported by a NHMRC Practitioner fellowship. P.G.E. is supported by a National Health and Medical Research Council (Australia) Clinical Research Fellowship (546244).

\section{References}

Avenanti, A., \& Aglioti, S. M. (2006). The sensorimotor side of empathy for pain. In M. Mancia (Ed.), Psychoanalysis and neuroscience (pp. 235-256). Milan: Springer.

Avenanti, A., Bueti, D., Galati, G., \& Aglioti, S. M. (2005). Transcranial magnetic stimulation highlights the sensorimotor side of empathy for pain. Nature Neuroscience, 8, 955-960. doi:10.1038/ nn1481

Avenanti, A., Minio-Paluello, I., Bufalari, I., \& Aglioti, S. M. (2006). Stimulus-driven modulation of motor-evoked potentials during observation of others' pain. NeuroImage, 32, 316-324. doi:10.1016/j.cortex.2008.10.004

Avenanti, A., Minio-Paluello, I., Bufalari, I., \& Aglioti, S. M. (2009a). The pain of a model in the personality of an onlooker: Influence of state-reactivity and personality traits on embodied empathy for pain. NeuroImage, 44, 275-283. doi:10.1016/j. neuroimage.2008.08.001

Avenanti, A., Minio-Paluello, I., Sforza, A., \& Aglioti, S. M. (2009b). Freezing or escaping? Opposite modulations of empathic reactivity to the pain of others. Cortex, 45, 1072-1077. doi:10.1016/j. cortex.2008.10.004

Banissy, M. J., Walsh, V. Z., \& Muggleton, N. G. (2011). Mirror-touch synaesthesia: A case of faulty self-modelling and insula abnormality. Cognitive Neuroscience, 2, 114-115.

Banissy, M. J., \& Ward, J. (2007). Mirror-touch synesthesia is linked with empathy. Nature Neuroscience, 10, 815-816. doi:10.1038/ nn1926

Baron-Cohen, S., \& Wheelwright, S. (2004). The empathy quotient: An investigation of adults with Asperger syndrome or high functioning autism, and normal sex differences. Journal of Autism and Developmental Disorders, 34, 163-175. doi:10.1023/B: JADD.0000022607.19833.00

Beck, A. T., Ward, C. H., Mendelson, M., Mock, J., \& Erbaugh, J. (1961). An inventory for measuring depression. Archives of General Psychiatry, 4, 561-571.

Blakemore, S. J., Bristow, D., Bird, G., Frith, C., \& Ward, J. (2005). Somatosensory activations during the observation of touch and a case of vision-touch synaesthesia. Brain, 128, 1571-1583. doi:10.1093/brain/awh500

Botvinick, M., Jha, A. P., Bylsma, L. M., Fabian, S. A., Solomon, P. E., \& Prkachin, K. M. (2005). Viewing facial expressions of pain engages cortical areas involved in the direct experience of pain. NeuroImage, 25, 312-319. doi:10.1016/j.neuroimage.2004.11.043

Bufalari, I., Aprile, T., Avenanti, A., Di Russo, F., \& Aglioti, S. M. (2007). Empathy for pain and touch in the human somatosensory cortex. Cerebral Cortex, 17, 2553-2561. doi:10.1093/cercor/ bhl161

Carlsson, K., Petrovic, P., Skare, S., Petersson, K. M., \& Ingvar, M. (2000). Tickling expectations: Neural processing in anticipation 
of a sensory stimulus. Journal of Cognitive Neuroscience, 12, 691-703. doi:10.1162/089892900562318

Carr, L., Iacoboni, M., Dubeau, M., Mazziotta, J. C., \& Lenzi, G. (2003). Neural mechanisms of empathy in humans: A relay from neural systems for imitation to limbic areas. Proceedings of the National Academy of Sciences, 100, 5497-5502. doi:10.1073/ pnas.0935845100

Cheng, Y., Yang, C., Lin, C., Lee, P., \& Decety, J. (2008). The perception of pain in others suppresses somatosensory oscillations: A magnetoencephalography study. NeuroImage, 40, 1833-1840. doi:10.1016/j.neuroimage.2008.01.064

Coghill, R. C., McHaffie, J. C., \& Yen, Y. F. (2003). Neural correlates of interindividual differences in the subjective experience of pain. Proceedings of the National Academy of Sciences, 100, 85388542. doi:10.1073/pnas.1430684100

Davis, M. H. (1980). A multidimensional approach to individual differences in empathy. JSAS Catalog of Selected Documents in Psychology, 10, 85.

Decety, J., \& Jackson, P. L. (2004). The functional architecture of human empathy. Behavioral and Cognitive Neuroscience Reviews, 3, 71-100. doi:10.1177/1534582304267187

Derbyshire, S. W. G., Jones, A. K. P., Gyulaic, F., Clark, S., Townsend, D., \& Firestone, L. L. (1997). Pain processing during three levels of noxious stimulation produces differential patterns of central activity. Pain, 73, 431-445. doi:10.1016/S0304-3959(97)00138-3

de Vignemont, F., \& Singer, T. (2006). The empathic brain: How, when and why? Trends in Cognitive Sciences, 10, 435-441. doi:10.1016/j.tics.2006.08.008

di Pellegrino, G., Fadiga, L., Fogassi, L., Gallese, V., \& Rizzolatti, G. (1992). Understanding motor events: A neurophysiological study. Experimental Brain Research, 91, 176-180.

Enticott, P. G., Johnston, P. J., Herring, S. E., Hoy, K. E., \& Fitzgerald, P. B. (2008). Mirror neuron activation is associated with facial emotion processing. Neuropsychologia, 46, 2851-2854. doi:10.1016/j.neuropsychologia.2008.04.022

Enticott, P. G., Kennedy, H. A., Bradshaw, J. L., Rinehart, N. J., \& Fitzgerald, P. B. (2010). Understanding mirror neurons: Evidence for enhanced corticospinal excitability during the observation of transitive but not intransitive hand gestures. Neuropsychologia, 48, 2675-2680. doi:10.1016/j.neuropsychologia.2010.05.014

Fadiga, L., Craighero, L., \& Olivier, E. (2005). Human motor cortex excitability during the perception of others' action. Current Opinion in Neurobiology, 15, 213-218. doi:10.1016/j.conb.2005.03.013

Farina, S., Tinazzi, M., Le Pera, D., \& Valeriani, M. (2003). Painrelated modulation of the human motor cortex. Neurological Research, 25, 130-142. doi:10.1179/016164103101201283

Fecteau, S., Pascual-Leone, A., \& Theoret, H. (2008). Psychopathy and the mirror neuron system: Preliminary findings from a nonpsychiatric sample. Psychiatry Research, 160, 137-144. doi:10.1016/j.psychres.2007.08.022

Fitzgerald, P. B., Fountain, S., \& Daskalakis, Z. J. (2006). A comprehensive review of the effects of rTMS on motor cortex excitability and inhibition. Clinical Neurophysiology, 117, 2584-2596. doi:10.1016/j.clinph.2006.06.712

Fitzgibbon, B. M., Enticott, P. G., Giummarra, M. J., Thompson, R., Georgiou-Karistianis, N., \& Bradshaw, J. L. (2011). Atypical electrophysiological activity in amputees who experience synaesthetic pain when observing pain in another. Social Cognitive and Affective Neuroscience. doi:10.1093/scan/nsr016

Fitzgibbon, B. M., Enticott, P. G., Rich, A., Giummarra, M. J., Geogiou-Karistianis, N., \& Tsao, J. W. (2010a). High incidence of 'synaesthesia for pain' in amputees. Neuropsychologia, 48, 3675-3678. doi:10.1016/j.neuropsychologia.2010.07.029

Fitzgibbon, B. M., Giummarra, M. J., Georgiou-Karistianis, N., Enticott, P. G., \& Bradshaw, J. L. (2010b). Shared pain: From empathy to synaesthesia. Neuroscience and Biobehavioural Reviews, 34, 500 512. doi:10.1016/j.neubiorev.2009.10.007

Gallese, V., Fadiga, L., Fogassi, L., \& Rizzolatti, G. (1996). Action recognition in the premotor cortex. Brain, 119, 593-609. doi:10.1093/brain/119.2.593

Gangitano, M., Mottaghy, F. M., \& Pascual-Leone, A. (2001). Phasespecific modulation of cortical motor output during movement observation. NeuroReport, 12, 1489-1492. doi:10.1097/ 00001756-200105250-00038

Gazzola, V., Aziz-Zadeh, L., \& Keysers, C. (2006). Empathy and the somatotopic auditory mirror system in humans. Current Biology, 16, 1824-1829. doi:10.1016/j.cub.2006.07.072

Giummarra, M. J., \& Bradshaw, J. L. (2008). Synaesthesia for pain: Feeling pain with another. In J. A. Pineda (Ed.), Mirror Neuron Systems: The role of mirroring processes in social cognition (pp. 287-307). Totowa, NJ: Humana Press.

Giummarra, M. J., Gibson, S. J., Fitzgibbon, B. M., GeorgiouKaristianis, N., Nicholls, M. E. R., \& Bradshaw, J. L. (2010). Ouch! My leg jumps when you stab "my" hand. Perception, 39, 1396-1407. doi:10.1068/p6582

Godinho, F., Magnin, M., Frot, M., Perchet, C., \& Garcia-Larrea, L. (2006). Emotional modulation of pain: Is it the sensation or what we recall? Journal of Neuroscience, 26, 11454-11461. doi:10.1523/JNEUROSCI.2260-06.2006

Goller, A. I., Richards, K., Novak, S., \& Ward, J. (2011). Mirror-touch synaesthesia in the phantom limbs of amputees. Cortex. doi:10.1016/j.cortex.2011.05.002

Haker, H., \& Rossler, W. (2009). Empathy in schizophrenia: Impaired resonance. European Archives of Psychiatry and Clinical Neuroscience, 259, 352-361. doi:10.1007/s00406-009-0007-3

Han, S., Fan, Y., \& Mao, L. (2008). Gender differences in empathy for pain: An electrophysiological investigation. Brain Research, 1196, 85-93. doi:10.1016/j.brainres.2007.12.062

Haraldsson, H. M., Ferrarelli, F., Kalin, N. H., \& Tononi, G. (2004). Transcranial magnetic stimulation in the investigation and treatment of schizophrenia: A review. Schizophrenia Research, 71, 116.

Iannetti, G. D., \& Mouraux, A. (2010). From the neuromatrix to the pain matrix (and back). Experimental Brain Research, 205(1), 112.

Jackson, P. L., Meltzoff, A. N., \& Decety, J. (2005). How do we perceive the pain of others? A window into the neural processes involved in empathy. NeuroImage, 24, 771-779.

Jarvelainen, J., Schurmann, M., \& Haria, R. (2004). Activation of the human primary motor cortex during observation of tool use. NeuroImage, 23, 187-192. doi:10.1016/j. neuroimage.2004.06.010

Kaplan, J. T., \& Iacoboni, M. (2006). Getting a grip on other minds: Mirror neurons, intention understanding, and cognitive empathy. Social Neuroscience, 1, 175-183.

Keysers, C., Wicker, B., Gazzola, V., Anton, J., Fogassi, L., \& Gallese, V. (2004). A touching sight: SII/PV activation during the observation and experience of touch. Neuron, 42, 335-346. doi:10.1016/S0896-6273(04)00156-4

Kraskov, A., Dancause, N., Quallo, M. M., Shepherd, S., \& Lemon, R. (2009). Corticospinal neurons in macaque ventral premotor cortex with mirror properties: A potential mechanism for action suppression? Neuron, 64, 922-930. doi:10.1016/j.neuron.2009.12.010

Krings, T., Naujokat, C., \& von Keyserlingk, D. G. (1998). Representation of cortical motor function as revealed by stereotactic transcranial magnetic stimulation. Electroencephalography and Clinical Neurophysiology, 109, 85-93. doi:10.1016/S0924-980X (97)00078-7

Lamm, C., Decety, J., \& Singer, T. (2011). Meta-analytic evidence for common and distinct neural networks associated with directly 
experienced pain and empathy for pain. NeuroImage, 54, 2492 2502. doi:10.1016/j.neuroimage.2010.10.014

Lawrence, E. J., Shaw, P., Baker, D., Baron-Cohen, S., \& David, A. S. (2004). Measuring empathy: Reliability and validity of the Empathy Quotient. Psychological Medicine, 34, 911-924. doi:10.1017/S0033291703001624

Lepage, J. F., Tremblay, S., \& Theoret, H. (2010). Early non-specific modulation of corticospinal excitability during action observation. European Journal of Neuroscience, 31, 931-937. doi:10.1111/ j.1460-9568.2010.07121.x

Le Pera, D., Graven-Nielsen, T., Valeriani, M., Oliviero, A., Di Lazzaro, V., \& Tonali, P. A. (2001). Inhibition of motor system excitability at cortical and spinal level by tonic muscle pain. Clinical Neurophysiology, 112, 1633-1641. doi:10.1016/S13882457(01)00631-9

Lorenz, J., Minoshima, S., \& Casey, K. L. (2003). Keeping pain out of mind: The role of the dorsolateral prefrontal cortex in pain modulation. Brain, 126, 1079-1091. doi:10.1093/brain/awg102

Minio-Paluello, I., Avenanti, A., \& Aglioti, S. M. (2006). Left hemisphere dominance in reading the sensory qualities of others' pain? Social Neuroscience, 1, 320-333. doi:10.1080/17470910601035954

Minio-Paluello, I., Baron-Cohen, S., Avenanti, A., Walsh, V., \& Aglioti, S. M. (2009). Absence of embodied empathy during pain observation in Asperger syndrome. Biological Psychiatry, 65, 5562. doi:10.1016/j.biopsych.2008.08.006

Morrison, I., Lloyd, D., di Pellegrino, G., \& Roberts, N. (2004). Vicarious responses to pain in anterior cingulate cortex: Is empathy a multisensory issue? Cognitive, Affective, \& Behavioral Neuroscience, 4, 270-278. doi:10.3758/CABN.4.2.270

Morrison, I., Poliakoff, E., Gordon, L., \& Downing, P. (2007). Response-specific effects of pain observation on motor behavior. Cognition, 104, 407-416. doi:10.1016/j.cognition.2006.07.006

Newman-Norlund, R. D., Ganesh, S., van Schie, H. T., De Bruijn, E. R. A., \& Bekkering, H. (2009). Self-identification and empathy modulate error-related brain activity during the observation of penalty shots between friend and foe. Social Cognitive and Affective Neuroscience, 4, 10-22. doi:10.1093/scan/nsn028

Nicolson, S. E., Caplan, J. P., Williams, D. E., \& Stern, T. A. (2009). Comorbid pain, depression, and anxiety: Multifaceted pathology allows for multifaceted treatment. Harvard Review of Psychiatry, 17, 407-420. doi:10.3109/10673220903463226

Osborn, J., \& Derbyshire, S. W. G. (2010). Pain sensation evoked by observing injury in others. Pain, 148, 268-274.

Peyron, R., Laurent, B., \& Garcia-Larrea, L. (2000). Functional imaging of brain responses to pain: A review and meta-analysis. Clinical Neurophysiology, 30, 263-288. doi:10.1016/S0987-7053(00)00227-6

Pfeifer, J. H., Iacoboni, M., Mazziotta, J. C., \& Dapretto, M. (2008). Mirroring others' emotions relates to empathy and interpersonal competence in children. NeuroImage, 39, 2076-2085. doi:10.1016/j.neuroimage.2007.10.032

Ploghaus, A., Becerra, L., Borras, C., \& Borsook, D. (2003). Neural circuitry underlying pain modulation: Expectation, hypnosis, placebo. Trends in Cognitive Sciences, 7, 197-200. doi:10.1016/ S1364-6613(03)00061-5

Porro, C. A., Cettolo, V., Francescato, M. P., \& Baraldi, P. (2003). Functional activity mapping of the mesial hemispheric wall during anticipation of pain. NeuroImage, 19, 1738-1747. doi:10.1016/S1053-8119(03)00184-8

Porro, C. A., Martinig, M., Facchin, P., Maieron, M., Jones, A. K. P., \& Fadiga, L. (2007). Parietal cortex involvement in the localization of tactile and noxious mechanical stimuli: A transcranial magnetic stimulation study. Behavioural Brain Research, 178, 183-189. doi:10.1016/j.bbr.2006.11.011

Rainville, P. (2002). Brain mechanisms of pain affect and pain modulation. Current Opinion in Neurobiology, 12, 195-204.
Rizzolatti, G., \& Craighero, L. (2004). The mirror-neuron system. Annual Review of Neuroscience, 27, 169-192.

Rizzolatti, G., Fogassi, L., \& Gallese, V. (2001). Neurophysiological mechanisms underlying the understanding and imitation of action. Nature Reviews Neuroscience, 2, 661-670. doi:10.1038/ 35090060

Salanova, V., Andermann, F., Rasmussen, T., Olivier, A., \& Quesney, L. F. (1995). Parietal lobe epilepsy: Clinical manifestations and outcome in 82 patients treated surgically between 1929 and 1988 . Brain, 118, 607-627. doi:10.1093/brain/118.3.607

Schmidt, S., Cichy, R. M., Kraft, A., Brocke, J., Irlbacher, K., \& Brandt, S. A. (2009). An intial transient-state and reliable measures of corticospinal excitability in TMS studies. Clinical Neurophysiology, 120, 987-993. doi:10.1016/j.clinph.2009.02.164

Singer, T., Seymour, B., O’Doherty, J., Kaube, H., Dolan, R. J., \& Frith, C. D. (2004). Empathy for pain involves the affective but not sensory components of pain. Science, 303, 1157-1162. doi:10.1126/science. 1093535

Spielberger, C. D., Gorsuch, R. L., \& Lushene, R. E. (1970). Manual for the state-trait anxiety inventory. Palo Alto, CA: Consulting Psychologists Press.

Sullivan, M. J. L., Bishop, S. R., \& Pivik, J. (1995). The pain catastrophizing scale: Development and validation. Psychological Assessment, 7, 524-532.

Svensson, P., Miles, T. S., McKay, D., \& Ridding, M. C. (2003). Suppression of motor evoked potentials in a hand muscle following prolonged painful stimulation. European Journal of Pain, 7, 55-62. doi:10.1016/S1090-3801(02)00050-2

Urban, P. P., Solinski, M., Best, C., Rolke, R., Hopf, H. C., \& Dieterich, M. (2004). Different short-term modulation of cortical motor output to distal and proximal upper-limb muscles during painful sensory nerve stimulation. Muscle \& Nerve, 29, 663-669. doi:10.1002/mus.20011

Urgesi, C., Moro, V., Candidi, M., \& Aglioti, S. M. (2006). Mapping implied body actions in the human motor system. Journal of Neuroscience, 26, 7942-7949. doi:10.1523/JNEUROSCI.128906.2006

Van Damme, S., Crombez, G., \& Lorenz, J. (2007). Pain draws visual attention to its location: Experimental evidence for a threat-related bias. Journal of Pain, 8, 976-982. doi:10.1016/ j.jpain.2007.07.005

Vargas, C. D., Olivier, E., Craighero, L., Fadiga, L., Duhamel, J. R., \& Sirigu, A. (2004). The influence of hand posture on corticospinal excitability during motor imagery: A transcranial magnetic stimulation study. Cerebral Cortex, 14, 1200-1206. doi:10.1093/cercor/bhh080

Verhoeven, K., Crombez, G., Eccleston, C., Van Ryckeghem, D. M. L., Morley, S., \& Van Damme, S. (2010). The role of motivation in distracting attention away from pain: An experimental study. Pain, 149, 229-234. doi:10.1016/j.pain.2010.01.019

Wager, T. D., Rilling, J. K., Smith, E. E., Sokolik, A., Casey, K. L., \& Davidson, R. J. (2004). Placebo-induced changes in fMRI in the anticipation and experience of pain. Science, 303, 1162-1167. doi:10.1126/science. 1093065

Wicker, B., Keysers, C., Plailly, J., Royet, J. P., Gallese, V., \& Rizzolatti, G. (2003). Both of us disgusted in my insula: The common neural basis of seeing and feeling disgust. Neuron, 40, 655-664.

Wood, R., Gallese, V., \& Cattaneo, L. (2010). Visuotactile empathy within the primary somatosensory cortex revealed by shortlatency afferent inhibition. Neuroscience Letters, 473, 28-31. doi:10.1016/j.neulet.2010.02.012

Yang, C., Decety, J., Lee, S., Chen, C., \& Cheng, Y. (2009). Gender differences in the mu rhythm during empathy for pain: An electroencephalographic study. Brain Research, 1251, 176-184. doi:10.1016/j.brainres.2008.11.062 